\title{
A Contribution to the Automatic Measurement of X-Ray Diffraction Lines
}

\author{
Frank Henschel ${ }^{1} \&$ Jürgen Bauch ${ }^{1}$ \\ ${ }^{1}$ Institute for Material Science, Dresden University of Technology, Dresden, Germany \\ Correspondence: Jürgen Bauch, Institute for Material Science, Dresden University of Technology, Dresden \\ 01062, Germany. Tel: 49-351-4633-3594. E-mail: juergen.bauch@tu-dresden.de
}

Received: October 8, 2012 Accepted: April 10, 2013 Online Published: April 23, 2013

doi:10.5539/ijc.v5n2p47 URL: http://dx.doi.org/10.5539/ijc.v5n2p47

\begin{abstract}
In order to harness the full potential of X-ray diffraction methods, such as the high precision KosseL and X-ray Rotation Tilt (XRT) technique, a fast and automatic detection and evaluation process for digital recordings or digitized film is required. The presented method facilitates, through the implementation of largely automated processes, rapid access to a diverse evaluable database of many X-ray diffraction images. The method used for the detection of conic shaped diffraction reflection lines is composed of two parts: the rough detection of the desired image objects followed by the subsequent sub-pixel modeling of reflection lines. On the one hand the resulting database can as a whole be used for the quantitative analysis of material science-based basic phenomena with the aim of associating reflection abnormalities to crystallographic defects. On the other hand, due to the registration of the reflection fine structure, the individual data set is especially suitable for the direct calculation and output of precision residual stress tensors. For this application it is necessary to know the complete recording geometry. In this contribution we examine the focal curves for this purpose. These can also be used to evaluate electron backscatter diffraction pattern.
\end{abstract}

Keywords: X-ray microdiffraction, KosSEL technique, XRT technique, EBSD technique, residual stress

\section{Introduction}

Over the past several years the quality of digital X-ray recordings has improved enormously. Currently, further research is being undertaken in this direction as well as inevitably in the field of automated procedures and programs to interpret the available digital image information. In our group such procedures are of great interest for the analysis of diffraction patterns generated during the investigation of material's crystallographic structure, especially for the KosseL and the X-ray Rotation Tilt technique (XRT) (Bauch, 2002). For these techniques it is necessary to very accurately detect and evaluate the diffraction information, which comes in the form of conic sections. Up to now, a suitable process derived from a combination of different techniques has been developed and used (Bauch et al., 2011). It consists of two main parts: the detection of the generated objects followed by a sub-pixel modeling of the reflection lines. Firstly a three-dimensional HougH transform is used from which many initial imprecise object parameters are determined. On the basis of this approximate description a second subsequent step is undertaken in which the real and exact object parameters are calculated and fit into a geometric model. With these results, other image and lattice parameters respectively can be determined. The data obtained with this method from a single recording are well suited for the calculation of the precision strain tensor that can be transferred using the elastic constants in the residual stress tensor. This method is particularly well suited to the high accuracy of the Kossel technique. In addition, due to this new quantitative evaluation there is the potential to make systematic analysis of material science based basic phenomena, such as smeared or double reflection maxima and local maxima along a reflection, and to assign it to crystallographic effects.

\section{Method}

For the described application based exemplary on the two mentioned micro-diffraction methods: the KosSELand XRT-technique, a multifunction KosSEL-EBSD-SEM system and a self-development of the X-ray Rotation Tilt system (XRT) was used. For the mapping of Kossel lines a focused electron beam or collimated X-ray beam (diameter $<5 \mu \mathrm{m}$ or $<100 \mu \mathrm{m}$ ) generate within the interaction volume from a single grain of the sample, characteristic or fluorescence X-rays. Parts of the characteristic radiation and the total bremsstrahlung leave the crystal un-diffracted. This leads to undesirable film blackening. Only a small part of the characteristic X-rays are 
diffracted by the interference-capable lattice planes according to the BRAGG equation. These interferences fall on straight circular cones with half apex angles of $90^{\circ}-\Theta_{\mathrm{hkl}}\left(\Theta_{\mathrm{hkl}}\right.$ BRAGG angle) and create curves of $2^{\text {nd }}$ order (Kossel lines). Curves of the same order are generated using the XRT technique which is based on the same physical law; between each monochromatic X-ray beam with wavelength $\lambda$ and the lattice planes there exists an angle $\Theta_{\mathrm{hkl}}$ that satisfies the BRAGG equation. This leads to the occurrence of an interference point on a detector. Variation over the entire angular range in the half-space above the specimen produces a sequence of pixels on the detector (Figure 1). On the whole these result in joined diffraction lines generated from their different respective lattice planes. The various advantages and disadvantages of the two methods are explained in more detail by Bauch et al. (2003). However, both use the same method of analysis of the diffraction lines, which is divided into two necessary main parts: first a rough detection followed by a fine detection with sub-pixel accuracy. This subdivision is necessary in order to achieve automation on the one hand and on the other to utilize the high accuracy of the recording. The analysis is described in detail in a paper by Bauch et al. (2011), however, in summary the analysis is done by the way of this procedure: the first step of the object detection is the image conversion to a binary image via an edge detection algorithm like the SOBEL operator. The proper recognition of objects is based on the HougH transform. This is a robust method to detect simple geometric objects such as straight lines described for instance by Russ (2002). It is also possible to use this procedure for more complex geometric objects, but these results for every unknown parameter in an extra dimension of the relevant dual HoUGH-space solution and also in an unacceptable increase in computing time. The applied simplification for the first rough approximation is the use of circles with three unknown parameters (circle-center with pixel coordinates $\mathrm{x}$ and $\mathrm{y}$, and radius $\mathrm{r}$ ). For each positive pixel from the previously obtained binary output image a circle with corresponding coordinates for this center and also variable radius $r$ is drawn in the appropriate level $r$ of the three-dimensional solution space (Figure 2). By taking the HougH solution space as a multidimensional matrix, the cells with the highest accumulated values correspond to the solutions for centers and radius for the circles. For another way to reduce the parameters for HougH detection see in a paper by Maurice and Fortunier (2008), but without the following subsequent step: On the basis of this rough solution, sufficient transverse profiles from the diffraction lines along the circular paths are measured. Finally, these profile values form the basis for the approximating function, determined by adjustment calculus. Thereby, the conic sections are determined by using very high precision (see Figure 1 where an example of three superimposed curves of the detected reflections is shown).

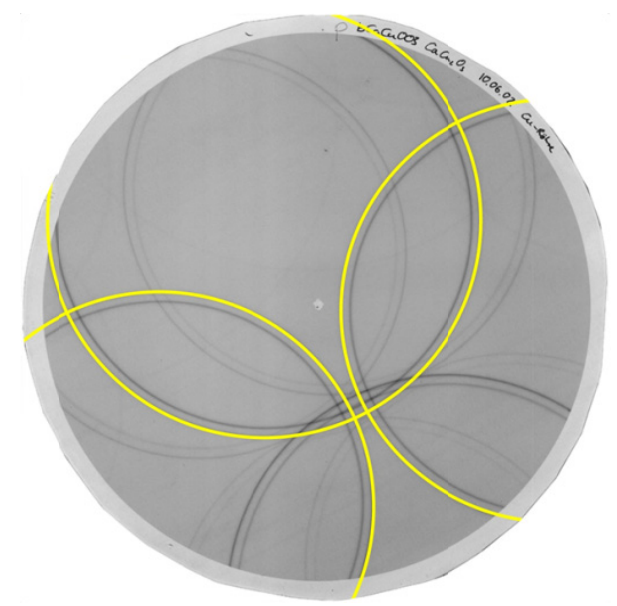

Figure 1. XRT diffraction image of a $\mathrm{CaCu}_{2} \mathrm{O}_{3}$ sample with three marked diffraction lines (conic sections) 


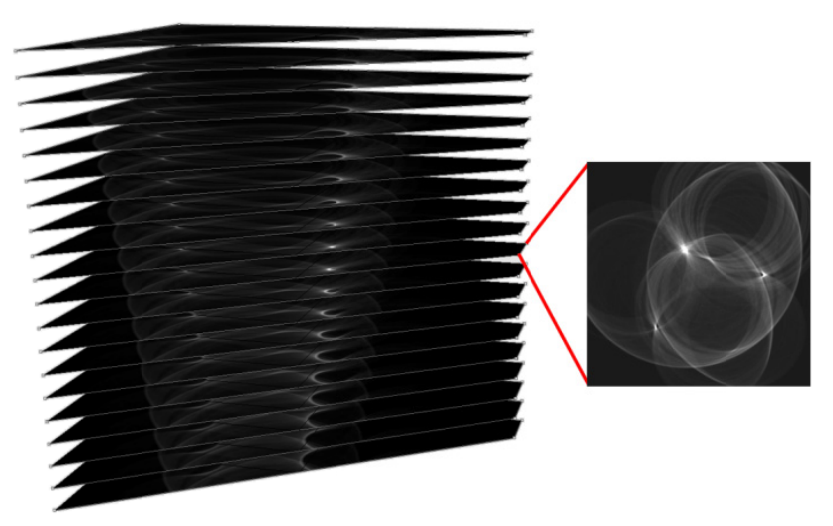

Figure 2. Intersection of three-dimensional HougH solution space with one single layer corresponding to the radius, with accumulated values and three maxima corresponding to the center of the circle. Thereafter, by using the fine structure, the conic sections are calculated (see Figure 1)

One result of this evaluation process is a complete parameterization of the conic sections. For further analysis, additional recording parameters are required: for example, the orthogonal projection of the apex of the generating cone (principal point). In a XRT-diffraction image (see Figure 1), this point may be already marked. Usually, the determination with required accuracy is very difficult. The result of this work is a new method to determine the parameters of the recording geometry, see Chapter 3.

\section{Results}

An exact description of the various diffraction lines of a recording can be used in various tasks and investigations. It permits amongst other things easier access to a detailed examination of the fine structure. For example, this can be extracted as an overall profile of the entire curve length, or a large number of transverse profiles (see profiles Figure 4 from diffraction line (202) in Kossel image Figure 3). The latter results combined a movie, which visualizes a cycle along a reflection. Consequently, anomalies in the fine structure like smeared or double maxima can be better identified. Through the investigation of a large number of samples it can be possible to assign these irregularities in the reflections to the corresponding crystallographic origins.
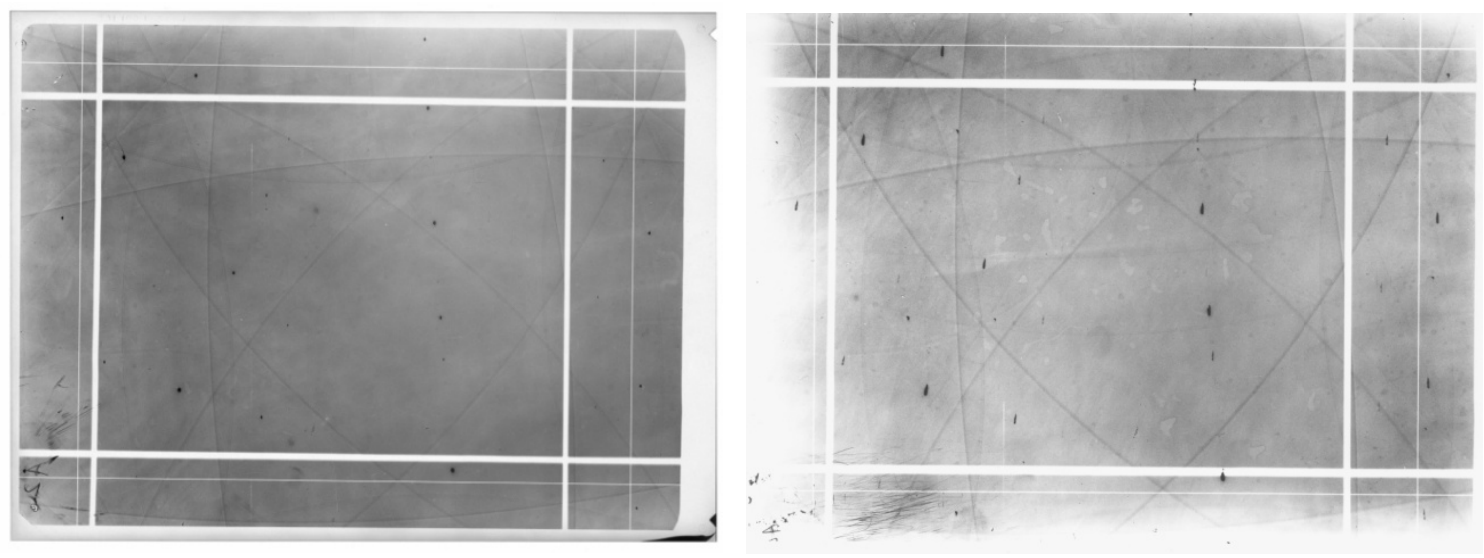

Figure 3. Two Synchrotron KosseL images of a series of recordings of CuMn4 with projection of the two level rectangular wire grid and with different residual stresses of the $3^{\text {rd }}$ kind 

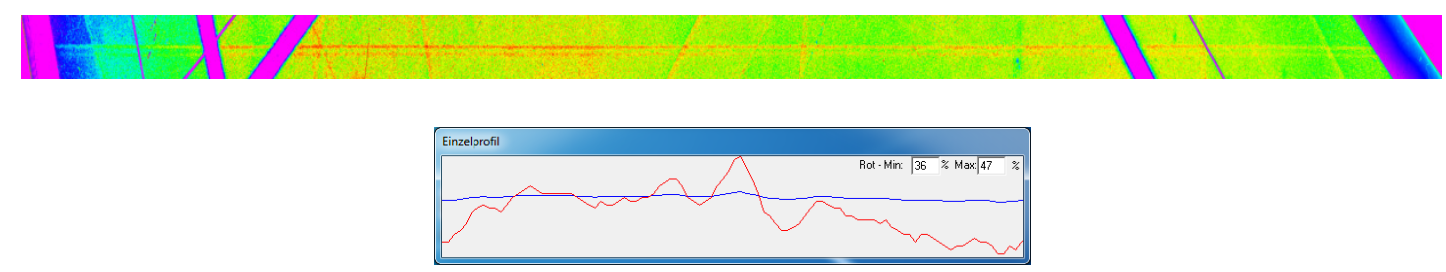

Figure 4. a) Profile along the diffraction line (202) of CuMn4 with visible intensity variation globally and locally (tone corrected HSV color space) (above); b) Single transverse profile (below)

From the data other important parameters can be derived: the high precision determination of the crystallographic orientation, lattice parameters and the strain (stress) tensor. The data generated from the KosSEL technique is suitable for these computations, due to its very high precision. This permits the analysis of residual stresses of the $3^{\text {rd }}$ kind. In a first step particular distances must be measured between the reflection lines or to a common point, to determine the strain tensor from the geometry of the recording (Bauch et al., 2004). An important point in the record data, which is suitable for this, is the principal point (gnomonic projection center). This together with the distance of the detector to the sample (focal distance) forms the interior orientation of the recording. In a recording made using the XRT technique the principal point is always identical to the image center and already marked on the detector. But usually these parameters are not known because every sample has different dimensions and is always fitted different in the sample chamber of the KosseL system too. With larger recording distances larger variations occur in the detector plane. There are several different ways to calculate the necessary complete orientation of this perspective. At first an additional construction formed from a rectangular wire grid at two different depth levels was built into the beam path (Figure 3). By using a defined parallel assembly (wire grid) to the detector plane the interior orientation can be calculated from the shadow projection by elementary mathematics (intercept theorem), as well as the relative position of the imaging sample point (exterior orientation). As a result of our work we have determined the necessary required accuracy of such a two-level target and the exact installation cannot be guaranteed. So that a more complex calculation is necessary: the spatial resection (Bonitz, 2009). This can be realized by an adjustment of the fit of the spatial control points to their image point. However, it makes more sense to determine the overall orientation from the diffraction pattern itself. There are several ways for this new approach: The individual reflection lines are all created equal: many circular diffraction cones with different axial directions and different apertures but common apex intersect with a single plane (image plane). Since there are no special cases, these sections are hyperbolas or ellipses. Due to the common apex all major axis regardless of the conic type intersect at one point. This is the perpendicular of the projection center (the apex; restricted sample area) to the detector plane: the definition of the principal point. In this analysis, the distance from the image plane to the sample is still required. This can be determined using another way without the use of any scaling factor only from the reflection lines. For every single conic there exists a family of cones with their apex on a special curve: the focal curve (Albrecht, 1994). For an ellipse it is a focal-hyperbola and vice versa: for a hyperbola a focal-ellipse. They lie in perpendicular planes to each other and the foci of one are the vertexes of the other (Figure 5). In addition, all focal curves from the diffraction image intersect at one point: the apex from the all KosseL-cones, the investigated point of the sample (Figure 6). Consequently, all the apertures are known as well as the diffraction angles (BRAGG's law). 


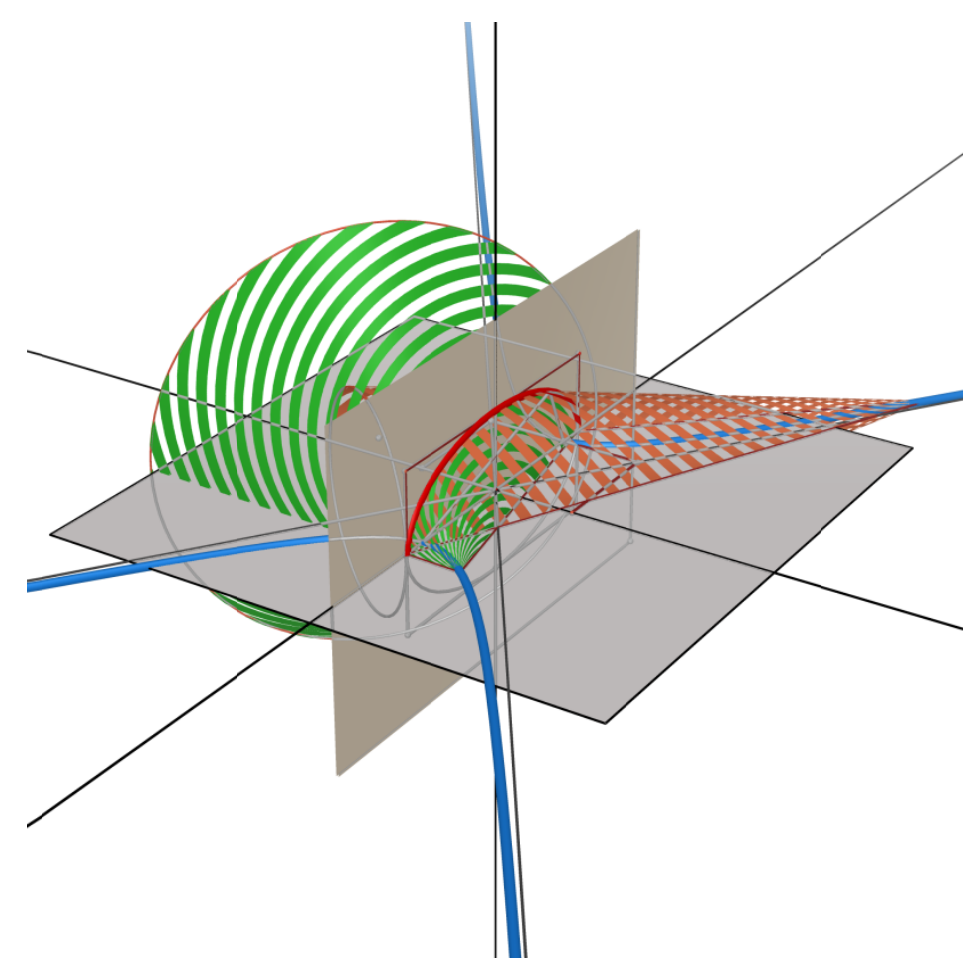

Figure 5. Ellipse and hyperbola in perpendicular planes where each form the focal curve of the other. Shown is an example of two right circular cones with their apex on the plotted hyperbola. These create the original ellipse

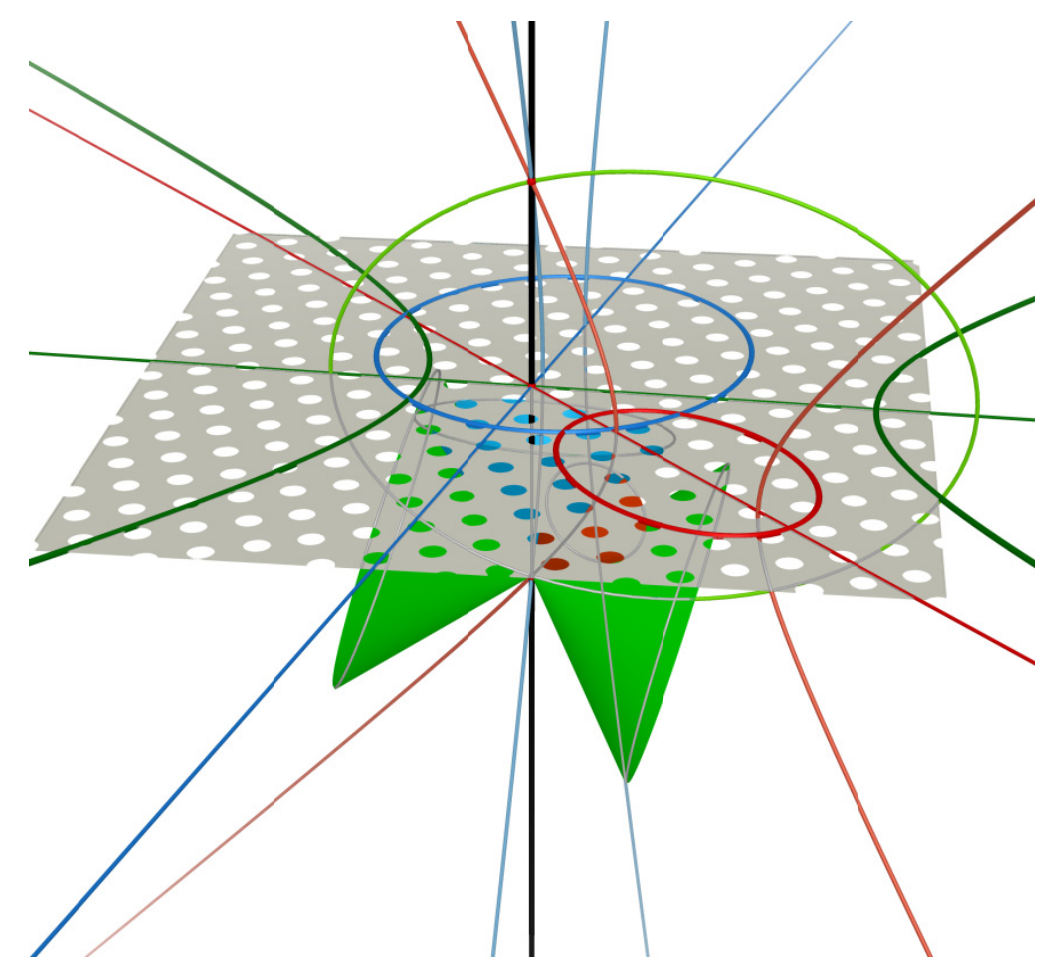

Figure 6. Three examples of different diffraction reflections in the detector plane (gray-with holes) with the respective focal curves (same color, lighter). The major axes intersect at the principal point and focal curves in the projection center, which is the apex of all diffraction cones-the sample (on both sides of detector plane, below the level the generating cones) 
This analysis shows that the record alone gives the full imaging geometry. In fact for the calculation of the existing approach a few steps are necessary. If the conic equations in the form $a x^{2}+b y^{2}+2 c x y+2 d x+2 e y+f=0$ are known after the detection process described by Bauch et al. (2011), then among others the principal axis transformation can be followed. By implementing other geometric tasks (intersection) computationally, the principal point can be obtained. Even at the highest precision in detecting the curves of the above-mentioned method, the results must be averaged over a remaining uncertainty. The use of focal curves however offers a completely new possibility to increase the accuracy. The adjustment calculus needs no longer to be limited to individual curves. It can be applied globally to the recording geometry with the fact that all diffraction cones have the same apex. As an example see Figure 7.

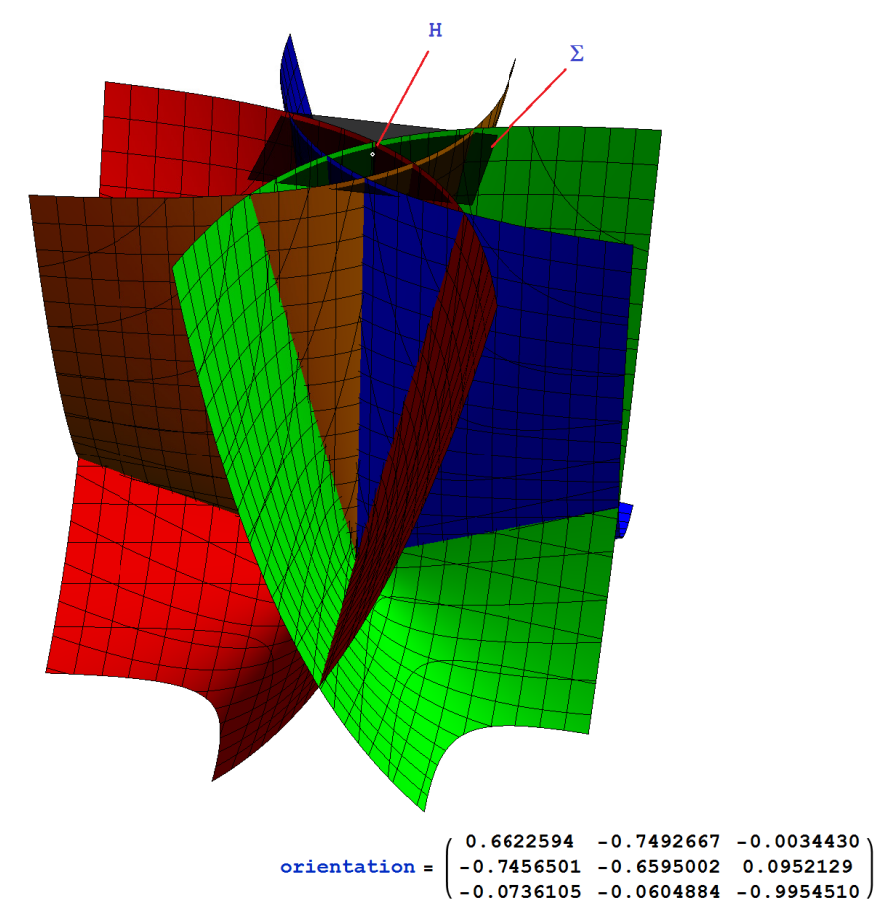

Figure 7. With use of focal curves, the complete orientation can be derived from the recording itself. The figure shows the real diffraction cones of the four reflection lines with indices (0-22), (022), (20-2), (202) and the image plane $\Sigma$ with the principal point H, see Figure 3: Synchrotron Kossel images of CuMn4. Contrary to Figure 6, the focal curves are not marked

The fact that all these procedures are computationally realizable, recordings can be evaluated automated and faster (orientation, precision lattice parameters etc.) such as in the XRT-recording of a $\mathrm{CaCu}_{2} \mathrm{O}_{3}$-sample, that crystallizes in the orthorhombic system (Figure 8). An extensive analysis from a LAUE exposure from the same sample would be simply because of the unknown wavelength very difficult. 


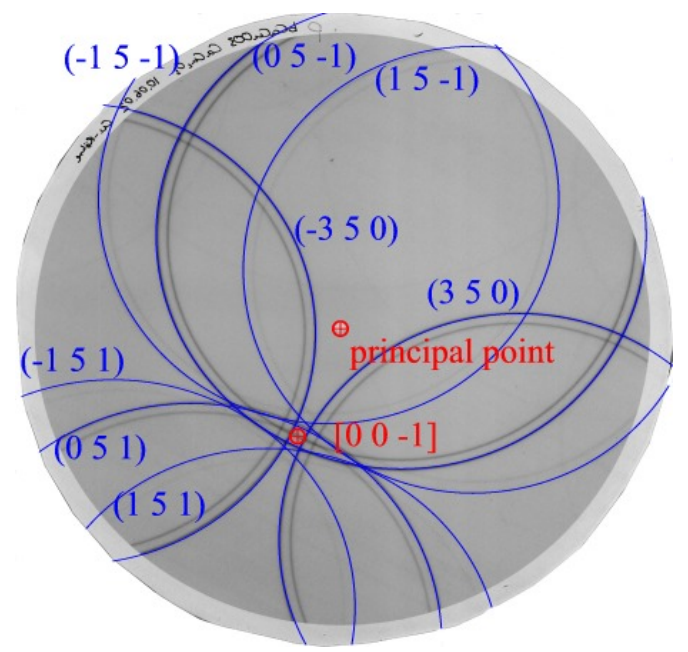

Figure 8. The same diffraction image as Figure 1 with marked principal point, Miller Indices and orientation

\section{Discussion}

The focus of this work was initially the very precise detection of $2^{\text {nd }}$ order curves in diffraction lines generated for example from the KOSSEL or XRT technique. From the outset it was important to make the method easy to use and mostly automated. As the accuracy of the KOSSEL technique is very suitable for the calculation of the strain tensor and residual stresses of the $3^{\text {rd }}$ kind, it is useful to optimize further for a good usability of analysis. There is no longer an extra object in the beam path necessary due to the imaging geometry with specimen-to-screen distance, principal point or pattern center can only be determined over the diffraction pattern themselves. In addition, the accuracy can be further increased as the measurement data are not optimized for individual curves, but for the entire recording geometry. That is useful for other diffraction imaging techniques such as EBSD, which are based on conic shaped diffraction pattern. In conclusion the presented method demonstrates a further step in the development of a high resolution "Residual stress microscope" and facilitates, through the implementation of largely automated procedures, a fast detection from diffraction lines and provides the complete recording geometry.

\section{Acknowledgements}

We are thankful for the financial support of the Deutsche Forschungsgemeinschaft. We also thank Dr. R. Boucher for fruitful discussions and his help.

\section{References}

Albrecht, G. (1994). Eine Bemerkung zum Satz von Ivory. Journal of Geometry, 50(1-2), 1-10. http://dx.doi.org/10.1007/BF01222657

Bauch, J. (2002). Habilitationsschrift, TU Dresden, Fakultät für Maschinenwesen.

Bauch, J., Ullrich, H. J., Böhling, M., \& Reiche, D. (2003). A comparison of the KosseL and the X-Ray Rotation-Tilt Technique. Cryst. Res. Technol., 38(6), 440-449.

Bauch, J., Wege, S., Böhling, M., \& Ullrich, H. J. (2004). Improved approaches to measurements of residual stresses in micro regions with the Kossel and the XRT technique. Cryst. Res. Technol., 39(7), 623-633

Bauch, J., Henschel, F., \& Schulze, M. (2011). Automatic detection and high resolution fine structure analysis of conic X-ray diffraction lines. Cryst. Res. Technol., 465, 450-454.

Bonitz, P. (2009). Freiformflächen in der rechnerunterstützten Karosseriekonstruktion und im Industriedesign. Springer-Verlag.

Maurice, C., \& Fortunier, R. (2008). A 3D Hough transform for indexing EBSD and KossEL patterns. Journal of Microscopy, 230, 3.

Russ, J. C. (2002). The image processing handbook. CRC Press. 